\title{
Twist springback measurement of AUV propeller blade based on profile deviation
}

\begin{abstract}
Geometrical error due to twist springback is one of major defects found in the near net or net shape part. It becomes more critical for complex part such as an Autonomous Underwater Vehicle (AUV) propeller blade. In this study, twist springback was quantified by comparing the blade and the die profiles obtained from commercial optical technique namely Alicona system. The results show a different pattern, where the twist springback becomes larger as the twist angle increases moving to the tip. The results show a strong agreement with the experiment.
\end{abstract}

Keyword: Cold forging; Profile; Propeller blade; Twist springback 